\title{
ECNU at SemEval-2016 Task 3: Exploring Traditional Method and Deep Learning Method for Question Retrieval and Answer Ranking in Community Question Answering
}

\author{
Guoshun Wu${ }^{1}$, Man Lan ${ }^{1,2 *}$ \\ ${ }^{1}$ Department of Computer Science and Technology, \\ East China Normal University, Shanghai, P.R.China \\ ${ }^{2}$ Shanghai Key Laboratory of Multidimensional Information Processing \\ 51141201064 decnu.cn, mlandes.ecnu.edu.cn*
}

\begin{abstract}
This paper describes the system we submitted to the task 3 (Community Question Answering) in SemEval 2016, which contains three subtasks, i.e., Question-Comment Similarity (subtask A), Question-Question Similarity (subtask B), and Question-External Comment Similarity (subtask C). For subtask A, we employed three different methods to rank question-comment pair, i.e., supervised model using traditional features, Convolutional Neural Network and Long-Short Term Memory Network. For subtask B, we proposed two novel methods to improve semantic similarity estimation between question-question pair by integrating the rank information of questioncomment pair. For subtask C, we implemented a two-step strategy to select out the similar questions and filter the unrelated comments with respect to the original question.
\end{abstract}

\section{Introduction}

The purpose of Community Question Answering task in SemEval 2016 (Nakov et al., 2016) is to provide a platform for finding good answers to new questions in a community-created discussion forum, where the main task (subtask C) is defined as follows: given a new question and a large collection of question-comment threads created by a user community, participants are required to rank the comments that are most useful for answering the new question. Obviously, this main task consists of two optional subtasks, i.e., Question-Comment Similarity (subtask A, also known as answer ranking), which is to re-rank comments/answers according to their relevance with respect to the question, and QuestionQuestion Similarity (i.e., subtask B, also known as question retrieval), which is to retrieve the similar questions according to their semantic similarity with respect to the original question.

To address subtask A, we explored a traditional machine learning method which uses multiple types of features, e.g., Word Match Features, Translationbased Features, and Lexical Semantic Similarity Features. Additionally, for subtask A, we also built a Convolutional Neural Network (CNN) model and a bidirectional Long Short-Term Memory (BLSTM) model to learn joint representation for questioncomment $(Q-C)$ pair. For subtask B, besides IR method and traditional machine learning method, we also proposed two novel methods to improve semantic similarity estimation between question-question $(Q-Q)$ pairs by integrating the rank information of $Q-C$ pairs. Since subtask $\mathrm{C}$ can be regarded as a joint work of the two above-mentioned subtasks, we implemented a two-step strategy to first select out similar questions and then to filter out the unrelated comments with respect to the original question.

The rest of this paper is organized as follows. Section 2 describes our system. Section 3 describes experimental setting. Section 4 and 5 report results on training and test sets. Finally, Section 6 concludes this work.

\section{System Description}

For subtask A, we presented three different method$\mathrm{s}$ i.e., using traditional linguistic features, learning a CNN model and a bidirectional LSTM model to represent question and comment sentences. For sub- 
task B, besides traditional methods, we proposed two novel methods to improve semantic similarity estimation between $Q-Q$ pairs by integrating the rank information of $Q-C$ pairs. The first is to adopt general ranking evaluation metrics of $Q_{0}-C$ and $Q_{1}-C$ (i.e., Spearman, Pearson, and Kendall Coefficient) as additional ranking scores or features of $Q_{0}-Q_{1}$ where $Q_{0}$ and $Q_{1}$ represent original question and its related question, respectively. The second is to extract features on $Q_{0}-C$ and $Q_{1}-C$ and to regard the cosine values which are calculated on these two feature vectors as additional features for $Q_{0}-Q_{1}$.

\subsection{Features Engineering}

All three subtasks can be regarded as an estimation task of sentence semantic measures which can be modeled by various types of features. In this work, we employed the following four types of features borrowed from previous work, i.e., Word Match Features, Translation Based Features, Topic Model Based Features, and Lexical Semantic Similarity Features. The details of these four types of features are described as follows. Note that the following four feature types are adopted in both $Q-Q$ and $Q-C$ pairs, here we took the $Q-Q$ pair for example.

Word Matching Feature (WM): This feature records the proportions of co-occurred words between a given sentence pair. Given a $Q-Q$ pair, this feature type is calculated using five measures: $\left|Q_{0} \cap Q_{1}\right|,\left|Q_{0} \cup Q_{1}\right| /\left|Q_{0}\right|,\left|Q_{0} \cap Q_{1}\right| /\left|Q_{1}\right|, \mid Q_{1}-$ $Q_{0}|/| Q_{1}|,| Q_{0}-Q_{1}|/| Q_{0} \mid$, where $\left|Q_{0}\right|$ and $\left|Q_{1}\right|$ denote the number of the words of $Q_{0}$ and $Q_{1}$.

Translation Based Feature (TB): The above WM feature only considers the overlapped words between $Q_{0}$ and $Q_{1}$ and thus it may fail to "bridge the lexical gap" between $Q-Q$ pair. One possible solution is to regard this task as a statistic machine translation problem between question and answer by using the IBM Model 1(Brown et al., 1993) to learn the word-to-word probabilities. Following (Xue et al., 2008; Surdeanu et al., 2011), we regarded $P\left(Q_{0} \mid Q_{1}\right)$, i.e., the translation probability of $Q_{1}$ when given $Q_{0}$, as a translation based feature. The probabilities are calculated as:

$$
\begin{gathered}
P\left(Q_{0} \mid Q_{1}\right)=\prod_{w \in Q_{0}} P\left(w \mid Q_{1}\right) \\
P\left(w \mid Q_{1}\right)=(1-\lambda) P_{t r}\left(w \mid Q_{1}\right)+\lambda P_{m l}(w \mid C) \\
P_{m l}\left(w \mid Q_{1}\right)=\sum_{a \in Q_{1}} P(w \mid a) P_{m l}\left(a \mid Q_{1}\right)
\end{gathered}
$$

where $P\left(Q_{0} \mid Q_{1}\right)$ is the probability that the $Q_{0}$ word $w$ is generated from $Q_{1}, \lambda$ is a smoothing parameter, $C$ is a background collection. $P_{m l}(w \mid C)$ is computed by maximum likelihood estimator. $P(w \mid a)$ denotes the translation probability from $Q_{1}$ word $a$ to $Q_{0}$ word $w$. The GIZA++ Toolkit ${ }^{1}$ is used to compute these probabilities.

Topic Model Based Feature (TMB): We used the LDA (Blei et al., 2003) model to transform $Q_{0}$ and $Q_{1}$ into topic-based vectors and then took the cosine value of two topic vectors as feature. We use the GibbsLDA++ (Phan and Nguyen, 2007) Toolkit to train the topic model.

Lexical Semantic Similarity Feature (LSS): Inspired by (Yih et al., 2013), we included the lexical semantic similarity features in our model. We used three different word vectors to represent LSS feature, i.e., the 300-dimensional version of word $2 \mathrm{vec}$ (Mikolov et al., 2013) vectors, 300-dimensional Glove vectors (Pennington et al., 2014) and 300dimensional vectors which are pre-trained with the unsupervised neural language model (Mikolov et al., 2013) on the Qatar Living data ${ }^{2}$. Words not present in the set of pre-trained words are initialized randomly. There are two ways to calculate the LSS features. One is to calculate the cosine similarity by summing up all word vectors in $Q_{0}$ and $Q_{1}$. Another is to adopt averaged pairwise cosine similarity between each word in $Q_{0}$ and $Q_{1}$.

Besides above four types of features, for $Q-Q$ pair, we also extracted following two question information features $(\mathbf{Q I})$ to describe the informativeness of related question $Q_{1}$ : (1) the number of words in $Q_{1}$ (2) the position of $Q_{1}$ in all related questions. For $Q-C$ pair, we also extracted following two comment information features (CI) to measure the informativeness of a comment text: (1) the number of words in comment (2) the number of nouns, verbs and adjectives in comment.

\subsection{Two Methods to address subtask A}

\subsubsection{Method 1: CNN}

We proposed a convolutional neural network to model question-comment sentence. As illustrated in

\footnotetext{
${ }^{1}$ http://www.statmt.org/moses/giza/GIZA++.html

${ }^{2}$ http://alt.qcri.org/semeval2015/task3/index.php?id=dataand-tools
} 
Figure 1, we first input word embeddings (here we used 300-dimensional Glove vectors in (Pennington et al., 2014)) of question and comment words and then learn the meaning (i.e., feature vector) of question and comment through convolution and pooling operation. After a simple concatenation layer connecting question and comment vectors, we final obtain a relevant score through a softmax operation.

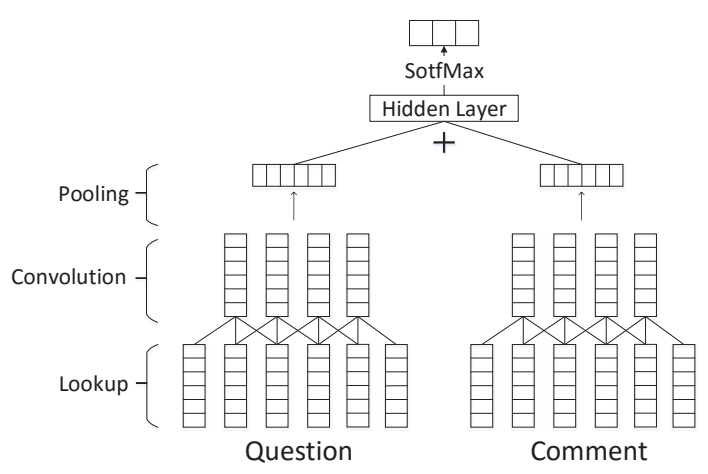

Figure 1: An illustration of CNN for question-comment similarity estimation.

\subsubsection{Method 2: BLSTM}

Figure 2 shows a multiple-layer BLSTM network model we used for question and comment sentences modeling. The procedure of BLSTM is similar to that of CNN. The words of question and comment sentences are first converted into vectors by looking up publicly available 300-dimensional Glove vectors. Then they are sequentially read by BLSTM from both directions. In this way, the contextual information across words in both question and comment sentences is modeled by employing temporal recurrence in BLSTM. Like CNN, finally it outputs a relevant score between question and comment by a simple concatenation operation on these two output vectors and a softmax operation.

\subsection{Two Methods for subtask B}

To calculate semantic similarity between $Q_{0}$ and $Q_{1}$ pair, previous work extracted features only from the $Q-Q$ sentence pair. We stated that the comment set $C$ and its rank with respect to $Q_{1}$ also provide useful information for question-question similarity. To address it, we propose two novel methods to improve

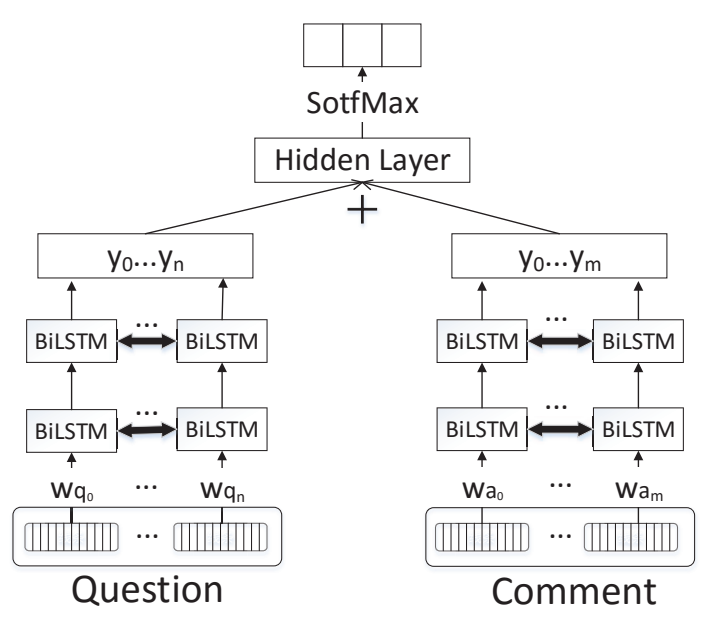

Figure 2: An illustration of BLSTM model for questioncomment similarity estimation.

semantic similarity estimation between $Q-Q$ pair by integrating the rank information of $Q-C$ pair.

\subsubsection{Method 1: adopt $Q-C$ Ranking Evaluation Metrics as Similarity Score}

The first method is to adopt rank evaluation metrics, i.e., Spearman, Pearson, and Kendall Ranking Coefficient directly as similarity scores for question similarity estimation.

Generally, these three nonparametric metrics are to measure the statistical dependence between two variables and to assess how similar between two variables. In comment ranking, they are used to measure how similar the two rankings $Q_{0}-C$ and $Q_{1}-C$ are. Based on our consideration, given one comment set $C$, if the two ranks of $Q_{0}-C$ and $Q_{1^{-}}$ $C$ are similar, the semantic similarity between $Q_{0^{-}}$ $Q_{1}$ is high. These three ranking correlation coefficients (i.e., Spearman, Pearson, and Kendall Coefficient) can be used directly as question similarity scores or used as additional ranking scores in combination with other features (described in Section 2.1) extracted from $Q_{0}-Q_{1}$ pair.

\subsubsection{Method 2: add new features extracted from $Q-C$ pair}

We presented two methods to add new features extracted from $Q-C$ pair. The first is to extract the features from $Q_{0}-C$ and $Q_{1}-C$ pair and then use the cosine scores calculated on the two feature vec- 
tors as additional features for $Q_{0}-Q_{1}$. We extracted traditional NLP features described in Section 2.1 from $Q_{0}-C$ and $Q_{1}-C$ pairs, respectively, denoted as two feature vectors, i.e., $F_{0}$ and $F_{1}$. Then we calculated the cosine similarity on these two vectors respectively, and obtain two cosine scores, i.e., $\cos \left(Q_{0}-C\right)$, and $\cos \left(Q_{1}-C\right)$. After that, we calculated the absolute difference between these two cosine scores. Finally, the obtained scores (denoted as $\left.\left[\cos _{1}, \cos _{2}, \ldots\right]\right)$ are ranked as additional features.

The second is to calculate the ranking scores of $Q_{0}-C$ and $Q_{1}-C$ by using comment ranking model firstly, then use the Manhattan Distance of two lists of ranked scores as an additional feature.

\subsection{A Two-Step Filtering for Subtask C}

To overcome the error propagation from questionquestion similarity step to question-comment similarity step, we employed a two-step filtering strategy for subtask C. The first step is to choose the top $N$ similar questions with the aid of the $Q-C$ ranking. The second step is to re-rank the comment and choose the top $M$ comments with integration of the previous $Q-Q$ results.

\section{Experimental Setting}

\subsection{Datasets}

Table 1 shows the statistics of training, development and test data sets, where the \#_original, \#_related, and \#_answers represent the number of original questions, related questions and answers, respectively. The types of comments with respect to original question and related question fall into three classes: Good, PotentiallyUseful and Bad. The types of related question with respect to original question fal1 into three classes: PerfectMatch, Relevant and Irrelevant.

\begin{tabular}{c|c|c|c|c}
\hline Subtask & Data & \#_original & \#_related & \#_answers \\
\hline \multirow{3}{*}{$\mathrm{A}$} & train & - & 5,898 & 37,848 \\
& dev & - & 500 & 5,000 \\
& test & - & 327 & 3,270 \\
\hline \multirow{3}{*}{ B } & train & 267 & 2,669 & 26,690 \\
& dev & 50 & 500 & 5,000 \\
& test & 70 & 700 & 7,000 \\
\hline \multirow{3}{*}{$\mathrm{C}$} & train & 267 & 2,669 & 26,690 \\
& dev & 50 & 500 & 5,000 \\
& test & 70 & 700 & 7,000 \\
\hline
\end{tabular}

Table 1: Statistics of datasets.

\subsection{Preprocessing}

We first removed stop words and punctuation, and changed all words to lowercase. After that, we performed tokenization and stemming using $\mathrm{NLTK}^{3}$ Toolkit.

\subsection{Evaluation Metrics}

To evaluate performance of the tasks, the Mean Average Precision (MAP) is adopted as official evaluation measure by the organizers which the MAP is defined as the mean of the averaged precision scores for queries.

\subsection{Learning Algorithm}

We compared two ranking strategies in traditional method. One is to train a pairwise-based ranking model, i.e., Learning-to-rank (Trotman, 2005), and use the output of model as a ranking score directly. Another is to first train a supervised classification model and then use the confidence score of probability as a ranking score. To train a supervised classifier, two algorithms implemented in SKLearn ${ }^{4}$ have been examined, i.e., Logistic Regression (LR) and Support Vector Machine (SVM). Finally, Logistic Regression classifier (penalized argument $c=1$ ) is adopted for all three subtasks for its good performance in preliminary experiments.

\section{Experiments on Training Data}

\subsection{Results on Subtask A}

For the experiments of subtask A, the hyperparameters of CNN model are set as follows: the number of filter windows is 2, feature maps are set to 100 , learning rate is set to 0.01 . And the hyperparameters of BLSTM model are set as follows: memory size is set to 500 and the learning rate is 0.01. Table 2 shows the results of subtask A with three different methods.

Firstly, all CI, TB, TMB, and LSS features significantly over WM baseline. Since CI is a measure of the informativeness of comment text, this indicates that users trend to choose the comment with more information. TB can learn word alignment between different words. Unlike the surface word matching features which only consider the surface word, the

\footnotetext{
${ }^{3}$ http://www.nltk.org/

${ }^{4} \mathrm{http}: / /$ scikit-learn.org/stable/
} 


\begin{tabular}{l|c|c}
\hline Methods & Features & MAP $(\%)$ \\
\hline & WM & 57.13 \\
Traditional & .+TB & 58.91 \\
NLP & .+TMB & 61.37 \\
Features & .CI & 63.03 \\
& .LSS & $\mathbf{6 5 . 3 7}$ \\
\hline CNN & - & 65.04 \\
\hline BLSTM & - & 65.13 \\
\hline Tra + CNN + BLSTM & - & $\mathbf{6 6 . 8 4}$ \\
\hline
\end{tabular}

Table 2: Results of subtask A using different methods. “..” means to add current feature to the previous feature set.

LSS features are obtained by integrating context of the word. Therefore, the LSS features show that this particular word embedding seems to complemen$t$ the surface word matching information. Secondly, the combination of five types of features achieve the best performance for traditional method. Thirdly, the model based CNN and BLSTM achieve comparable performance with traditional method. Finally, the combination of three methods achieve the best performance which shows that CNN and BLSTM catch complementary information for $Q-C$ pair with traditional method.

\subsection{Results on Subtask B}

Table 3 summarizes the results of subtask B with NLP features and integrating the rank information of $Q-C$ pair. Here Lucene represents using Lucene

\begin{tabular}{|c|c|c|}
\hline Method & Features & $\operatorname{MAP}(\%)$ \\
\hline Lucene & BM25 & 69.95 \\
\hline \multirow{5}{*}{$\begin{array}{l}\text { Traditional } \\
\text { NLP } \\
\text { Features }\end{array}$} & WM & 69.91 \\
\hline &.$+\mathrm{TB}$ & 70.72 \\
\hline &.$+\mathrm{TBM}$ & 71.05 \\
\hline &.$+\mathrm{LSS}$ & 72.13 \\
\hline &.$+\mathrm{QI}$ & 74.03 \\
\hline \multirow{6}{*}{ Method 1} & Pearson & 61.18 \\
\hline & Spearman & 62.86 \\
\hline & Kendall & 62.95 \\
\hline & Pearson $+N L P$ & 68.15 \\
\hline & Spearman $+N L P$ & 68.49 \\
\hline & Kendall $+N L P$ & 68.95 \\
\hline \multirow{3}{*}{ Method 2} & NLP & 74.03 \\
\hline &.$+\mathrm{ARC}$ & 74.25 \\
\hline & $+\mathrm{ARR}$ & 75.04 \\
\hline
\end{tabular}

Table 3: Results of subtask B using different methods.

Toolkit ${ }^{5}$ with the original question as query with BM25 $\left(K_{1}=1.2\right.$ and $\left.B=0.75\right) . A R C$ and $A R R$

\footnotetext{
${ }^{5}$ https://lucene.apache.org/
}

are the first and second methods presented in Section 2.3.2. According to the results of Table 3, we can make following three observations:

(1) Traditional NLP features significantly improve the performance of question-question similarity over Lucene baseline.

(2) The Pearson, Spearman, and Kendall get similar performance and do not perform well versus traditional NLP method. The three rank correlation$s$ all take down the performance of traditional NLP method when combined with it. The possible reason is that the ranked scores of comments are obtained by pre-trained comment ranking model which has a limitation of performance.

(3) Both $A R C$ and $A R R$ make contributions to the performance which means combining the information of $Q-C$ pair is helpful to find related questions.

\subsection{Results on Subtask C}

Table 4 depicts the results on subtask $\mathrm{C}$, where $W M Q, T M B Q$ and $T B Q$ represent extracting word matching, topic model based and translation based features on original question and related question. From Table 4, we observe the similar results with those in subtask A, i.e., traditional features make contribution to comment ranking. Moreover, the performance is improved by adding features extracted from $Q-Q$ pair, which indicates that the information extracted from $Q-Q$ pair makes significant contribution to answer ranking subtask.

\begin{tabular}{l|l|c}
\hline Method & Features & MAP(\%) \\
\hline Lucene & BM25 & 24.59 \\
\hline \multirow{3}{*}{ Traditional } & WM & 30.15 \\
NLP & .+CI & 33.13 \\
Features & .+TB & 34.27 \\
& .+TMB & 35.80 \\
& .+LSS & $\mathbf{3 6 . 8 2}$ \\
\hline \multirow{4}{*}{$Q-Q$ Features } & NLP & 36.82 \\
& .+WMQ & 38.14 \\
& .+TMBQ & 38.51 \\
& .+TBQ & $\mathbf{3 9 . 3 9}$ \\
\hline
\end{tabular}

Table 4: Results of subtask C with different traditional NLP features.

However, above results are evaluated using MAP on top 10 comments. Therefore the errors introduced in question retrieval (subtask B) would be prorogated to answer ranking (subtask A) and finally reduce the whole performance of CQA (subtask $\mathrm{C}$ ). 
To solve this problem, we investigated a two-step method to first filter unrelated comments and then filter unrelated answers. Figure 3 shows the results of two filtering methods on MAP metric, where $N$ represents the number of top related questions and $M$ represents the number of top ranked answers.
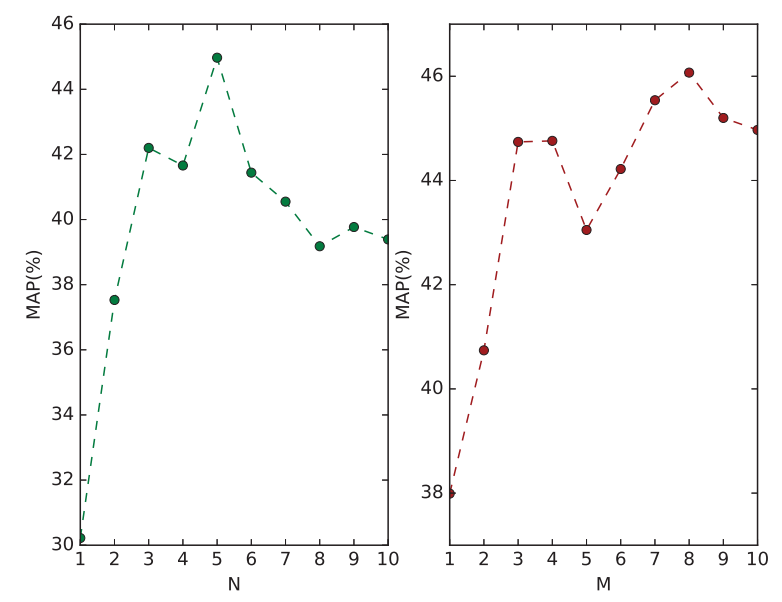

Figure 3: The results of subtask $\mathrm{C}$ using two-step filtering operation.

From left subplot of the Figure 3, we see that the best performance with filtering operation are much higher than the best score $(M A P=39.39 \%)$ without any filtering. The best performance $44.97 \%$ is obtained when $N=5$ and $M=10$. The reason may be that the filtering operation of unrelated questions can take away many unrelated comments for original question. The right subplot of the Figure 3 shows the performance curve $(N=5)$ when increasing the values of $M$. Similarly, the performance increases with $M$ increasing from 7 to 9 and it achieves the best score of $46.07 \%$ when $N=5$ and $M=8$.

\subsection{System Configuration}

Based on above experimental analysis, the three system configurations are as followings:

(1) subtask A: We used the combination of traditional method, CNN and BLSTM as the primary run in the test set. Traditional method and BLSTM serve as contrastive 1 run and contrastive 2 run.

(2) subtask B: Traditional method with Method 2 is used as primary run in the test set. The combination of traditional method with Method 2 and Lucene is contrastive 1 run and traditional method alone is contrastive 2 run.

(3) subtask C: The two-step filtering operation with $N=5$ and $M=8$ serves as primary run in the test set. The two-step filtering operation with $N=4$ and $M=7$ is contrastive 1 run. Traditional features adding $Q-Q$ pair information is used as contrastive2 run.

\section{Results on Test Data}

Table 5 shows the results on test set which are released by the organizers.

\begin{tabular}{c|c|c}
\hline subtask & run(rank) & MAP(\%) \\
\hline \multirow{4}{*}{ A } & ECNU-primary(4) & 77.28 \\
& ECNU-contrastive1 & 71.34 \\
& ECNU-contrastive2 & 75.71 \\
\cline { 2 - 3 } & Kelp-primary(1) & 79.19 \\
\hline \multirow{4}{*}{ B } & ECNU-primary(7) & 73.92 \\
& ECNU-contrastive1 & 73.25 \\
& ECNU-contrastive2 & 71.62 \\
\cline { 2 - 3 } & UH-PRHLT-primary(1) & 76.70 \\
\hline \multirow{4}{*}{$\mathrm{C}$} & ECNU-primary(7) & 46.47 \\
& ECNU-contrastive1 & 48.49 \\
& ECNU-contrastive2 & 47.24 \\
\cline { 2 - 3 } & SUper team-primary(1) & 55.41 \\
\hline
\end{tabular}

Table 5: Our results and the best results on three subtask test sets.

From the results, we find: (1) In subtask A, the combination of three methods significantly improve the performance over the traditional method and BLSTM, which is consistent with the results on training data as our expectation. (2) In subtask B, the result using traditional features is higher than Lucene but still has a certain gap with the best result. The possible reason may be because several traditional features do not work well in the test set. (3) In subtask C, beyond our expectation, the method using two-step filtering operation does not make obvious contribution. The possible reason may be that the values of $M$ and $N$ are not suitable for test set.

\section{Conclusion}

In this paper, we proposed multiple strategies (i.e., traditional method of extracting features and deep learning models) to address Community Question Answering task in SemEval 2016. For subtask A, we trained a classifier and learned the questioncomment representation based CNN and BLSTM. The combination of three models obtains the best 
results. For subtask B, we proposed two novel methods to improve semantic similarity estimation between $Q-Q$ pairs by utilizing the information of $Q-C$ ranking. For subtask $\mathrm{C}$, we employed a two step filtering strategy to reduce the noise which taking from unrelated comments. The results on test set show the effectiveness of our methods.

\section{Acknowledgments}

This research is supported by grants from Science and Technology Commission of Shanghai Municipality (14DZ2260800 and 15ZR1410700), Shanghai Collaborative Innovation Center of Trustworthy Software for Internet of Things (ZF1213).

\section{References}

David M Blei, Andrew Y Ng, and Michael I Jordan. 2003. Latent dirichlet allocation. the Journal of machine Learning research, 3:993-1022.

Peter F. Brown, Vincent J. Della Pietra, Stephen A. Della Pietra, and Robert L. Mercer. 1993. The mathematics of statistical machine translation: Parameter estimation. Comput. Linguist., 19(2):263-311, June.

Tomas Mikolov, Ilya Sutskever, Kai Chen, Greg S Corrado, and Jeff Dean. 2013. Distributed representations of words and phrases and their compositionality. In C.J.C. Burges, L. Bottou, M. Welling, Z. Ghahramani, and K.Q. Weinberger, editors, Advances in Neural Information Processing Systems 26, pages 3111-3119. Curran Associates, Inc.

Preslav Nakov, Lluís Màrquez, Walid Magdy, Alessandro Moschitti, Jim Glass, and Bilal Randeree. 2016. Semeval-2016 task 3: Community question answering. In Proceedings of the 10th International Workshop on Semantic Evaluation (SemEval 2016), Berlin, Germany, August. Association for Computational Linguistics.

Jeffrey Pennington, Richard Socher, and Christopher D. Manning. 2014. Glove: Global vectors for word representation. In Proceedings of the 2014 Conference on Empirical Methods in Natural Language Processing (EMNLP 2014), pages 1532-1543.

Xuan-Hieu Phan and Cam-Tu Nguyen. 2007. Gibbslda++: Ac/c++ implementation of latent dirichlet allocation (lda).

Mihai Surdeanu, Massimiliano Ciaramita, and Hugo Zaragoza. 2011. Learning to rank answers to nonfactoid questions from web collections. Computational Linguistics, 37(2).
Andrew Trotman. 2005. Learning to rank. Information Retrieval, 8(3):359-381.

Xiaobing Xue, Jiwoon Jeon, and W. Bruce Croft. 2008. Retrieval models for question and answer archives. In Proceedings of the 31st Annual International ACM SIGIR Conference on Research and Development in Information Retrieval, SIGIR '08, pages 475-482, New York, NY, USA. ACM.

Wen-tau Yih, Ming-Wei Chang, Christopher Meek, and Andrzej Pastusiak. 2013. Question answering using enhanced lexical semantic models. In Proceedings of the 51st Annual Meeting of the Association for Computational Linguistics (Volume 1: Long Papers), pages 1744-1753, Sofia, Bulgaria, August. Association for Computational Linguistics. 